\title{
Neues von den Freunden des Palmengartens e. V., Palmengarten-Gesellschaft
}

Wieder ist über ein erfolgreiches Jahr der Freunde des Palmengartens e. V. zu berichten. 2012 wurden für Einzel-Projekte (u.a. die Ausstattung der Grotte im Palmenhaus mit Aquarien, Fischen und Pflanzen, die Erstellung der Internetplattform „Garten“ sowie der Druck der Programme) rund $62500 €$ ausgegeben. Die regelmäßige Förderung (Führungen, Vorträge, Schülerseminar etc.) lag bei rund $22000 €$. Für 2013 ist die Förderung des Palmengartens mit rund $160000 €$ geplant.

Einziger Zweck der 1931 gegründeten Gesellschaft ist die Förderung des Palmengartens Frankfurt am Main. In der Regel erfolgt dies durch die (Mit-)Finanzierung von Projekten, die ohne diese Förderung nicht verwirklicht werden könnten. Neben Mitgliedsbeiträgen und Spenden wird inzwischen durch den persönlichen Einsatz von Mitgliedern, z. B. bei der Betreuung von Ausstellungen, diesem Bestreben weitere Nachhaltigkeit verliehen.

Die Gesellschaft hat, nach Bereinigung der Nichtzahler in 2011/2012, etwa 2200 Mitglieder. 2012 konnten 127 neue Mitglieder begrüßt werden. Erfreulich ist, dass auch wieder Jüngere den Weg in die Gesellschaft gefunden haben. 2012 sind durch Umzug, Tod und aus finanziellen Gründen 100 Personen ausgeschieden. Die regelmäßigen Vorstellungen der „Freunde“ mit einem Stand z. B. im Hessenpark Neu-Anspach anlässlich der Pflanzenbörsen, auf dem Fürstlichen Gartenfest in Schloss Wolfsgarten bei Langen, während Straßenfesten innerhalb der Stadt Frankfurt und bei Ausstellungen im Palmengarten zeigen unverändert Wirkung.

Der Anfang 2012 erfolgte Aufruf um Unterstützung in der Geschäftsstelle war erfolgreich. Mittlerweile hat ein Mitglied die Erledigung

Abb. 1: Der Vorstand. Von links nach rechts: Alexandra Prinzessin von Hannover, Herbert Billensteiner, Lolita Sebastian.

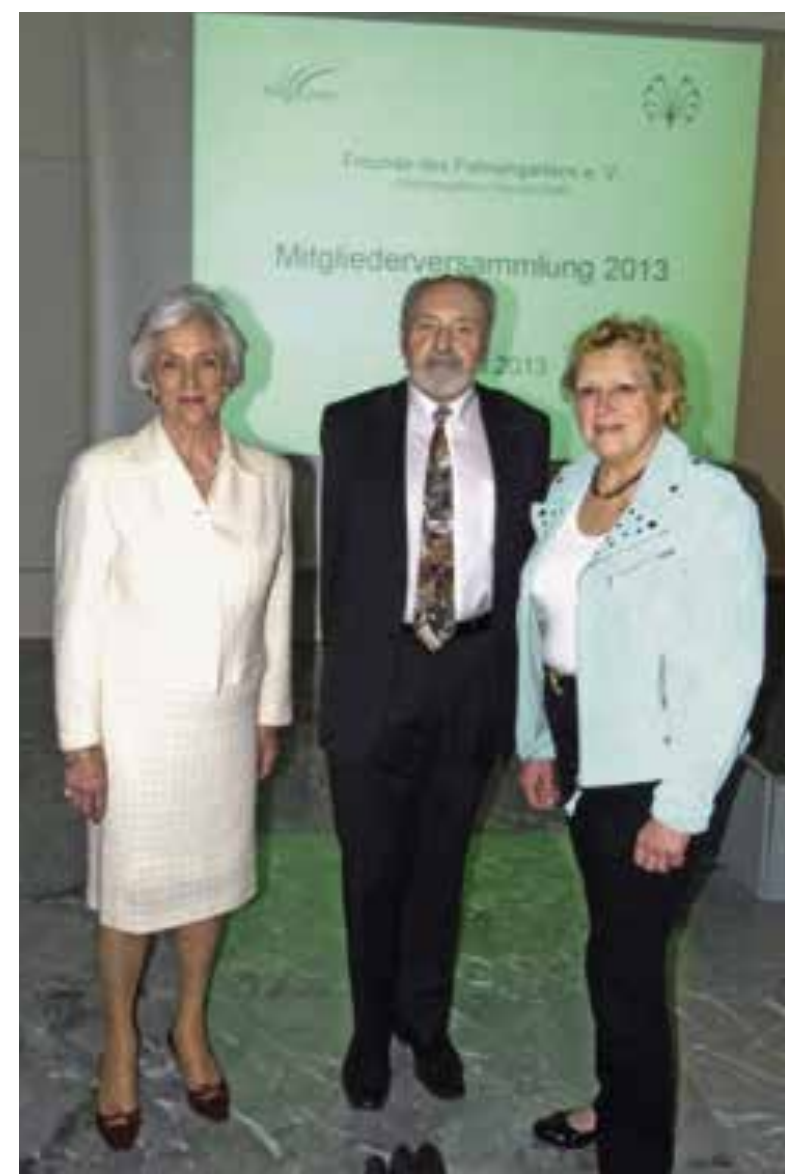

der Buchhaltung der Gesellschaft ehrenamtlich übernommen. Auch auf die Anfang 2013 an die Mitglieder ausgesprochenen Bitten um Unterstützung des Palmengartens bei Ausstellungen (vor allem Aufsicht) und Präsentationen kam positive Resonanz. Es zeigt sich, dass „das in Erinnerung rufen“ eine gute Möglichkeit ist, mehr Mitglieder in die Aktivitäten einzubinden. Der Dank des Vorstandes geht an alle ehrenamtlichen Helferinnen und Helfer, die sich während des Jahres mit nicht nachlassendem Einsatz für die „Freunde“ und den Palmengarten engagiert haben.

Neben zahlreichen Führungen und Vorträgen zu botanischen und gärtnerischen Themen wird jährlich ein Schülerseminar angeboten. Daneben gibt es von Reiseveranstaltern speziell für die Mitglieder ausgearbeitete Tages- und 


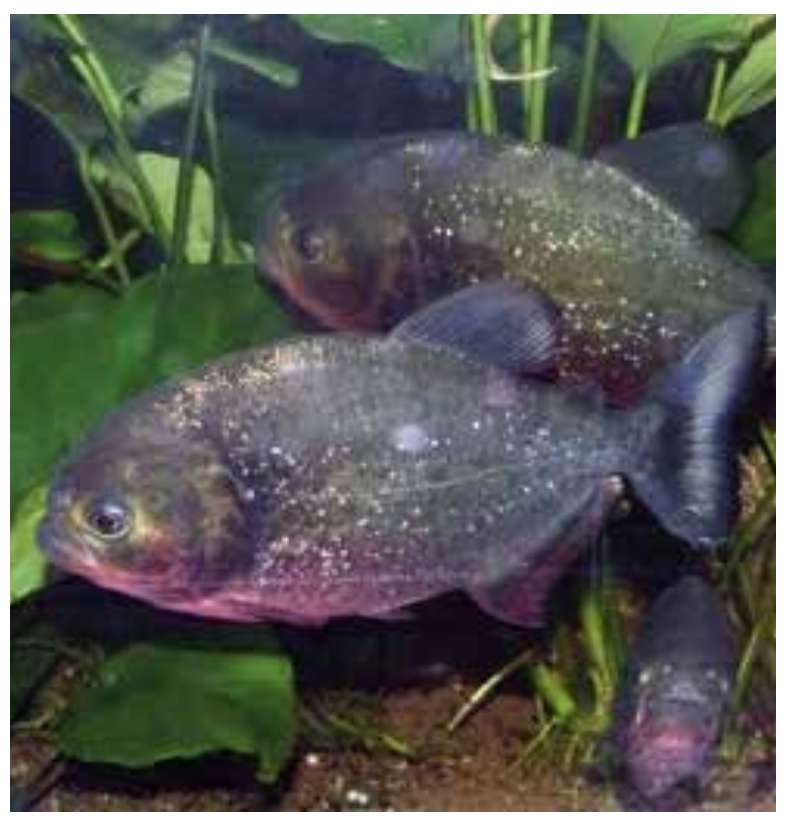

Mehrtagesreisen. Es ist Ziel, bei Mitgliedertreffen (so auch am 07. 11. 2012, als die "Freunde“ in den schönen Räumlichkeiten der KfW dort wiederum die Gastfreundlichkeit genießen durften) das Kennenlernen und den Austausch untereinander zu fördern. Kleine Gedichte, Musik und insbesondere der Bilderreigen durch 12 Monate im Palmengarten Frankfurt am Main von Hilke Steinecke rundeten das Treffen bei Kaffee und Kuchen ab.

Am 28. 11. 2012 hatten die Mitglieder die Möglichkeit, im restaurierten Gesellschaftshaus des Palmengartens den Fest- und Emporen-Saal sowie die Tagungsräume Orchidee und Lilie kostenlos zu besichtigen. Dies hat uns der Palmengarten durch Vermittlung von Matтhias Jenny ermöglicht. Auch die extra für uns geöffnete Grotte mit den von den „Freunden“ finanzierten neuen Aquarien sowie die Weihnachtsausstellung konnten bereits vor der offiziellen Eröffnung besichtigt werden. Die Anzahl der teilnehmenden Mitglieder (wir schätzen rund 350 - 400 Personen) war überwältigend.

Unser im Februar 2010 verstorbenes Mitglied Kamilla Nuyken hat die „Freunde“ mit einer Erbschaft bedacht. Daraus sind inzwischen $38700 €$ der Gesellschaft zugeflossen. Der Palmengarten hat zum Dank dafür nun eine Trauer-Ulme am Weg zwischen großem Weiher und Liegewiese gepflanzt. Derzeit wird überlegt, mit diesem Geld deutlich bestimmbare Projekte zu finanzieren und damit die Erblasserin entsprechend zu würdigen. An zusätzlichen Spenden aus den Mitgliedertreffen konnten in 2012 insgesamt 1 076,97 € (nach Abzug der Kosten) vereinnahmt werden. Auch hier geht der Dank des Vorstandes an die ehrenamtlichen Helfer, die zum Gelingen der Mitgliedertreffen beigetragen haben.

Die Mitgliederversammlung 2013 wurde am Mittwoch, den 17. April 2013 nach der fristund satzungsgemäß erfolgten Einladung durchgeführt. Die Vorsitzende, Alexandra PrinZESSIN VON HANNOVER, berichtete ausführlich über die Entwicklung der Mitgliederzahlen, Aktivitäten sowie die Förderung des Palmengartens. Dieser Bericht ist auch Grundlage für diesen Beitrag. Auf Antrag der Kassenprüferinnen wurde der Vorstand bei 3 Enthaltungen entlastet. Die bisherige Schatzmeisterin wurde mit 3 Nein-Stimmen und 13 Enthaltungen bei einer Gesamtpräsenz von 118 Mitgliedern für 5 Jahre wiedergewählt. Für 2013 ist anlässlich der Rosen-Ausstellung am Sonntag, den 9. Juni, wieder ein Mitgliedertreffen geplant. Gäste sind herzlich willkommen. Anmeldung in der Geschäftsstelle. Der Kostenbeitrag von $10 €$ pro Teilnehmer (keine Rückzahlung bei Nichtteilnahme) kann auf das Konto Nr. 27575605, BLZ 50010060 (Postbank Frankfurt) gezahlt werden.

Wir wünschen Ihnen allen weiterhin wunderschöne Aufenthalte im Palmengarten und gute Begegnungen mit den „Freunden“.

Der Vorstand, im April 2013

Abb. 2: Piranhas in einem der neuen Aquarien in der Grotte im Palmenhaus. 